\title{
Joining of Thin Films on Flexible Substrates with Nanosecond Laser Pulses
}

\author{
MARTIN EHRHARDT ${ }^{1}$, KLAUS ZIMMER ${ }^{* 1}$ \\ ${ }^{* 1}$ Leibniz-Institute of Surface Modification, Permoserstr. 15, 04318 Leipzig, Germany \\ E-mail: Klaus.zimmer@iom-leipzig.de \\ ${ }^{1}$ Leibniz-Institute of Surface Modification, Permoserstr. 15, 04318 Leipzig, Germany
}

\begin{abstract}
The joining of thin film components has been increasingly required for a variety of applications. In this presentation a novel laser joining process for bonding thin films was demonstrated. A $500 \mathrm{~nm}$ thick silver film on polyimide foil is bonded to a $12.5 \mu \mathrm{m}$ thick silver foil by laser exposure with $248 \mathrm{~nm}, 20 \mathrm{~ns}$ excimer pulses. The joining of the foil to the film is achieved at a laser fluence of $\sim 3.5 \mathrm{~J} / \mathrm{cm}^{2}$ with approx. 150 pulses. The surface morphology after rupture of the bonded silver areas was studied by SEM. A maximum failure shear force of $0.4 \mathrm{~N}$ was measured. DOI:10.2961/jlmn.2011.02.0003
\end{abstract}

Keywords: laser, thin films, joining, nanosecond, mechanical connection, welding

\section{Introduction}

The joining of thin film components has become progressively more important in an increasing number of applications. This has been of great interest especially in the fields of microtechnology, in which thin films and photovoltaic electrical or mechanical connections are of sizes in the sub-millimeter and micrometer range. However, the application of well-established techniques like wire bonding and soldering is limited either due to the need of specific metal layers or due to a sufficient film thickness. Laser joining techniques have a great potential due to, e.g., the focusing to spot size in the micrometer range for microjoining of thin film components. Classical laser processes for material joining have been developed mainly for steel and heavy industrial as well as for precision engineering. Typical techniques are soldering with and without additional materials and welding. Current studies aim at the development of bonding processes for thin metal films. However, due to the significant lower thicknesses of the films used, compared to traditional approaches and the usage of thermal sensitive substrates, e.g. polymers or semiconductor materials, new approaches have to be developed.

The damage thresholds for pulsed laser irradiation of thin metal films was studied by Matthias et al. with the result that the damage threshold decreases in accordance with a lower metal film thickness [1]. Typical types of damage are cracks, which may occur due to shockwaves during ablation, melting of the surface, or ablation at higher laser fluences in dependence on the thin film material properties, the adhesion to the substrate, and the properties of the substrate.

Once the laser fluence exceeds a certain threshold boiling and plasma processes occur. The relationship between vaporization of laser-heated metal and plasma formation is demonstrated in [2]. The generated plasma is capable of absorbing parts of the laser photons and causing laser beam absorption, which results in an attenuated beam at the metal surface. The basic processes involved in laser plasma absorption are discussed in [3].
In this study the joining of a thin film by pulsed laser radiation without auxiliary material is demonstrated. The laser bonding process was studied by SEM investigations and shear force measurements which showed the capabilities of this process.

\section{Experimental set-up and results}

For the experiments a $500 \mathrm{~nm}$ thick silver (Ag) film was deposited by magnetron sputtering on a polyimide substrate (UPILEX, $25 \mu \mathrm{m}$ ). An Ag foil was placed onto the Ag film. The Ag foil had a thickness of $12.5 \mu \mathrm{m}$ and was made by rolling (soft annealed). To fix the $\mathrm{Ag}$ foil to the top of the Ag film a vacuum chuck was used. Because the Ag foil was smaller than the Ag film, an additional polyimide foil which was larger than the $\mathrm{Ag}$ foil and the $\mathrm{Ag}$ film was used to fix the smaller $\mathrm{Ag}$ foil on top of the $\mathrm{Ag}$ film and to seal the whole vacuum chuck.

A KrF Excimer laser (LPX 220, Lambda Physik) with a pulse length of $t_{p}=20 \mathrm{~ns}$ and a wavelength of $\lambda=248 \mathrm{~nm}$ which is embedded in a laser workstation (Exitech, Ltd.) was used. Additionally, the workstation is comprised of a dielectric attenuator, beam shaping, and homogenizing optics. A Schwarzschild objective (15x demagnification) with an optical resolution of $1.5 \mu \mathrm{m}$ was used for projecting a variable aperture onto the sample. The laser spot size and the laser fluency were $100 \mu \mathrm{m} \times 100 \mu \mathrm{m}$ and $3.5 \mathrm{~J} / \mathrm{cm}^{2}$, respectively.

A rough estimation of the thermal diffusion length $\mathrm{L}_{\text {diff }}$ of the laser-induced heat can be done by the formula:

$$
L_{\text {diff }}=\sqrt{2 * \kappa * t_{\text {pulse }}}
$$

where $\kappa$ is the thermal diffusivity and $t_{\text {pulse }}$ the pulse duration. On the basis of this formula it can be seen that the heat penetration depth with the chosen pulse duration of the laser system is much less than with the normal used pulse duration in the $\mu \mathrm{s}$ and in the ms range for classical laser welding. Under this condition it can be assumed that the polyimide substrate of the samples is less influenced by the laser-induced heat. 
A computer-controlled laboratory tensile testing machine was used to measure the maximum shear force which could be applied on the join between the Ag foil and the Ag film. To adjust the forces to the testing range in the experiments at hand, the bonding area consisted of several joining points which were symmetrically arranged in three lines as sketched in figure 1 . The direction of tension caused by the tensile testing machine is indicated by the arrows in figure 1.

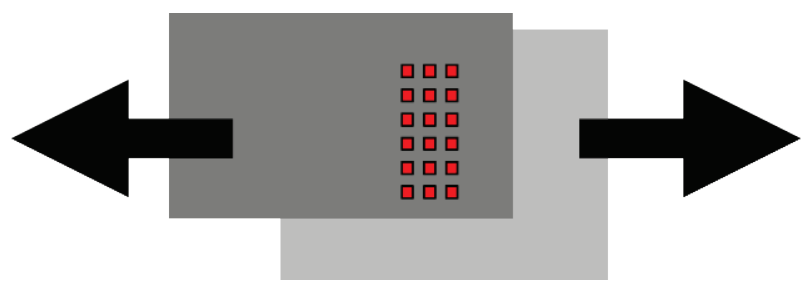

Fig. 1: Sketch of the joined Ag film (lower layer) with the Ag foil (upper layer). The red quadrates indicate the joining points. The arrows show the direction of tension which is induced due to the shear force measurement.

The total shear force, which can be loaded on one joining point until the joining point fails in dependence on the number of joining points per join, is shown in figure 2. Under the assumption that all joining points were equally loaded it can be concluded from figure 2 that the total failure shear force increases nearly linearly with the number of joining points per join. With 75 joining points a total shear force of $F_{\text {shear }}=0.47 \mathrm{~N}$ has to be loaded on the join before the join fails. The 75 joining points cover a calculated total joining area of $A=0.75 \mathrm{~mm}^{2}$. The resulting calculated total tensile strength of the join has a value of $\sigma_{\text {ten- }}$ sile $=0.63 \mathrm{~N} / \mathrm{mm}^{2}$. The calculated tensile strength of the other joins are in a similar range as it can be seen in figure 2, with the exception of the join consisting of 15 joining points which has a very weak joining strength. These values are much smaller than typical failure shear force values which were measured on metal-metal joins made with, e.g., laser welding, brazing, or bonding. However, it has to be kept in mind that none of these techniques can be applied to join metal films with a thickness of $500 \mathrm{~nm}$ on top of a flexible substrate.

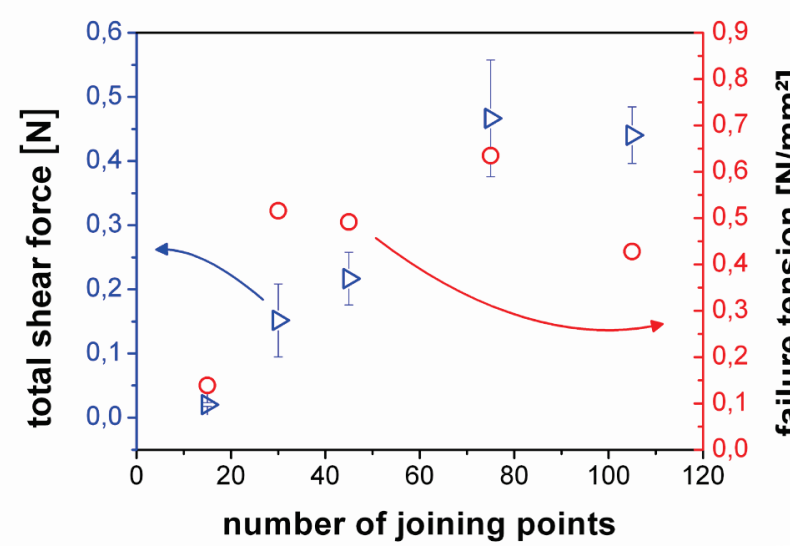

Fig. 2: Total shear force and the calculated failure tension in dependence on the number of joining points per join.
With the aim of examining the joining process and the failure after rupture SEM images of typical joining points after the shear force measurement were taken. Figure 3 shows SEM images of the laser-joined surfaces after rupture from the $\mathrm{Ag}$ film (figure $3 \mathrm{a}$ ) and the laser-irradiated $\mathrm{Ag}$ foil (figure $3 \mathrm{~b}$ ). In the images of the top Ag film, the imprint of the spot shape of the laser beam used is shown. This bulge in the size of the beam shape is caused by pressure forces to the silver foil that may result from various processes such as fast heating, material softening, thermal expansion, or pressures due to metal evaporation during the laser exposure of the Ag foil. Similar effects are known from laser shock peening [4].

Within the joined area of the Ag foil, parts of the silver film can be observed as depicted in figure 3a). These thin film pieces especially adhere to protuberances on the upper $\mathrm{Ag}$ foil. Furthermore, in figure 3a) it can also be seen that parts of the Ag film are lost and other parts are delaminated from the polyimide substrate due to the shear force measurement. Both caused the charging effects of the pure polyimide substrate as it is shown in the centre of the SEM image. These two observations give evidence for the main failure mechanisms of the joining points. The first failure mechanism is the exceeding of the maximum shear strength of the lower thin Ag film. The other main point which determines the strength of the joining points is the adhesive strength of the Ag film on the polyimide substrate.

No modifications outside of the area directly affected by the laser beam were detectable with the SEM as depicted in figure 3 . In addition, the polyimide substrate seems remarkably undamaged after the joining process also within the laser spot area.

No holes of the polyimide substrate were detectable by the SEM investigations. This is notable because the decomposition temperature of the polyimide substrate is near $550^{\circ} \mathrm{C}[5]$. This value is lower than the melting temperature of $\mathrm{Ag}\left(960^{\circ} \mathrm{C}[6]\right)$. That effect could explain that in the time scale of the used laser pulse duration the polyimide can be heated to a temperature higher than $550{ }^{\circ} \mathrm{C}[7,8]$.

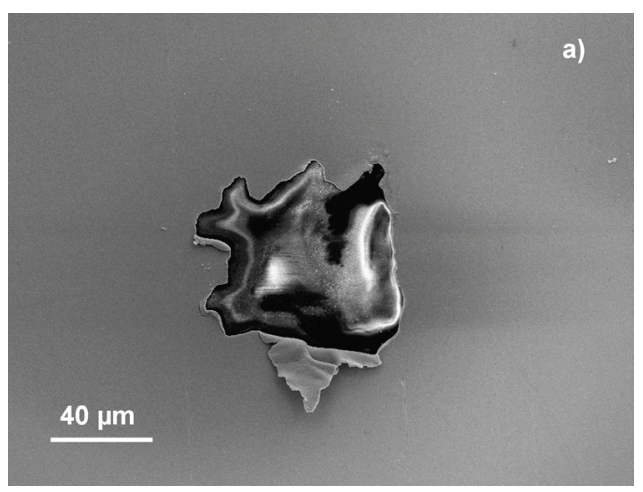




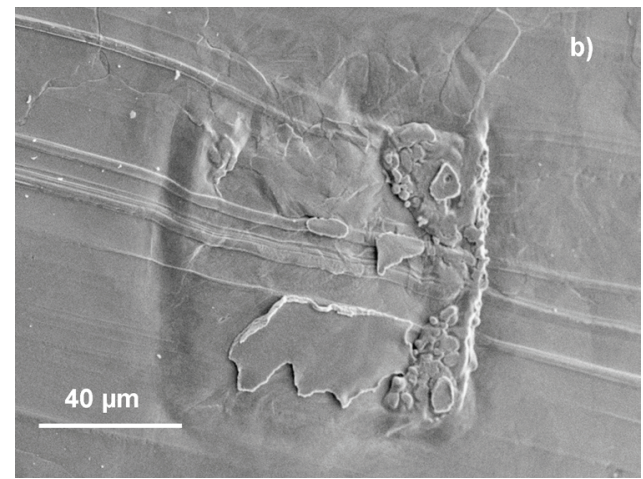

Fig. 3: SEM images of joining points after the failure shear force measurement; a) top view of the lower Ag film, b) lower side of the upper Ag foil. The selected joining points are representative; however, the joining point shown in a) is not the counterpart of the joining point in b).

\section{Conclusions}

Laser microjoining of thin silver films with thicknesses down to the sub-micrometer range have been demonstrated. The failure shear force of these joins can reach values of $0.47 \mathrm{~N}$ which corresponds to a failure tension of $\sigma_{\text {ten- }}$ sile $=0.63 \mathrm{~N} / \mathrm{mm}^{2}$.
The mechanical strength of the join between two Ag films can be improved by increasing the adhesion between the $\mathrm{Ag}$ film and the polyimide substrate. Investigations with the SEM show that the polyimide substrate is not damaged during the joining process.

\section{References}

[1] E. Matthias, M. Reichling, J. Siegel, O. W. Kading, S. Petzoldt, H. Skurk, P. Bizenberger, E. Neske: J. Appl. Phys. A, 58, (1994) 129.

[2] S. Petzoldt, J. Reif, E. Matthias: Appl. Surf. Sci., 96-8, (1996) 199.

[3] P. Mora: Phys. Fluids, 25, (1982) 1051.

[4] C. S. Montross, T. Wei, L. Ye, G. Clark, Y. W. Mai: Int. J. Fatigue, 24, (2002) 1021.

[5] S. Heltzel, C. O. A. Semprimoschnig: High Perform. Polym., 16, (2004) 235.

[6] J. S. Kim, T. Yokozuka, C. C. Lee: Mat. Sci. Eng. A, 458, (2007) 116.

[7] N. Arnold, N. Bityurin: Appl. Phys. A, 68, (1999) 615.

[8] H. Schmidt, J. Ihlemann, B. Wolff-Rottke, K. Luther, J. Troe: J. Appl. Phys., 83, (1998) 5458.

(Received: June 07, 2010, Accepted: April 02, 2011) 